University of Nebraska - Lincoln

DigitalCommons@University of Nebraska - Lincoln

Faculty Publications from the Department of Electrical \& Computer Engineering, Department Electrical and Computer Engineering

2011

\title{
A Directional Gamma Ray Detector Using a Single Chip Computational Sensor
}

\author{
Nathan R. Schemm \\ University of Nebraska-Lincoln, nathan.schemm@huskers.unl.edu \\ Sina Balkir \\ University of Nebraska-Lincoln, sbalkir2@unl.edu \\ Michael W. Hoffman \\ University of Nebraska-Lincoln, mhoffman1@unl.edu \\ Mark Bauer \\ University of Nebraska-Lincoln, mbauer2@unl.edu
}

Follow this and additional works at: https://digitalcommons.unl.edu/electricalengineeringfacpub

Part of the Electrical and Computer Engineering Commons

Schemm, Nathan R.; Balkir, Sina; Hoffman, Michael W.; and Bauer, Mark, "A Directional Gamma Ray Detector Using a Single Chip Computational Sensor" (2011). Faculty Publications from the Department of Electrical and Computer Engineering. 191.

https://digitalcommons.unl.edu/electricalengineeringfacpub/191

This Article is brought to you for free and open access by the Electrical \& Computer Engineering, Department of at DigitalCommons@University of Nebraska - Lincoln. It has been accepted for inclusion in Faculty Publications from the Department of Electrical and Computer Engineering by an authorized administrator of DigitalCommons@University of Nebraska - Lincoln. 


\title{
A Directional Gamma Ray Detector Using a Single Chip Computational Sensor
}

\author{
Nathan Schemm, Sina Balkır, Michael W. Hoffman, and Mark Bauer \\ University of Nebraska-Lincoln, \\ Department of Electrical Engineering, \\ 209N SEC, Lincoln, NE 68588-0511, USA \\ \{nathan.schemm@huskers.unl.edu, sbalkir@unl.edu,mhoffman1@unl.edu, mark@engr.unl.edu\}
}

\begin{abstract}
This paper presents the design and test results of a computational radiation sensor system based on a single chip solution that can determine the direction of gamma rays emitted from a radiation source. The overall system is formed by merging a sensor section with a compact and low power computational radiation sensor section. The sensor section houses three NaI gamma ray detectors arranged in a spatial configuration that allows for direction finding. The computational sensor is based on a single chip solution developed by authors that houses multiple low power sensor front ends, event driven analog-todigital converters, and a dedicated microcontroller on the same die. The presented system is capable of gathering the pulse height spectra from the gamma isotope data received from the three separate NaI detectors. Further processing of the data is possible by executing software algorithms using the computation resources available on chip. To that end, a compact fixed-point program was developed to perform on-chip real-time gamma ray collection and direction estimation. The single chip solution was fabricated in a $0.18 \mu \mathrm{m}$ CMOS technology with field tests demonstrating the validity of the approaches taken. The total computational sensor system power consumption is less than $20 \mu \mathrm{W}$, excluding the detector power consumption. The gamma isotope direction finding program executes in less than $1 \mathrm{~ms}$ with $5^{\circ}$ accuracy.
\end{abstract}

\section{INTRODUCTION}

The estimation of arrival angle for gamma rays emitted from isotopes is of great interest in radiation monitoring. In general, the existing capabilities of commercial off-the-shelf (COTS) solutions to detect isotopes are limited to hand-held units which mostly rely on a PDA or a portable PC platform to run analysis software [1], [2]. This limits the use of such systems for long term deployments due to their demanding power requirements in addition to their inherently bulky system configurations. Moreover, based on the characteristics of the leading detectors, isotope identification still remains as the main functionality, whereas the directional sensitivity is still an area under investigation.

Thus, a low-power and compact computational sensor paradigm that supports both gamma isotope identification and direction finding is highly desirable. Such a solution can lead to long term deployment scenarios for unattended radiation monitoring systems. The authors presented a compact computational sensor system for gamma isotope detection and identification in previous work [3]. The system utilized a custom low power microcontroller core, I/O hardware, timers, SRAM, and a bank of low power front ends on a single chip, designed and fabricated by authors using a $0.18 \mu \mathrm{m}$
CMOS technology [4], [5]. In this paper, the authors present the design and test of a directional gamma ray detector using the various resources available on the single chip solution. First, a sensor section has been constructed that houses three $\mathrm{NaI}$ gamma ray detectors arranged in a spatial configuration that allows for direction finding. Next, each $\mathrm{NaI}$ detector has been interfaced to a sensor front end, forming a three channel set up for individual monitoring of gamma ray activity in the detectors. Based on the individual activities and the properties of the spatial configuration of the detectors, a compact fixedpoint program was developed that runs on the chip for realtime gamma ray source direction estimation. Thus, the single chip solution leads to a fully functional radiation sensor with direction finding capability that is significantly lower power and smaller in size than an alternate COTS solution. In section II, the principles behind direction finding are presented. Section III presents the hardware design and implementation of the system. The embedded software development for direction finding is presented in section IV. Test results are presented in section V. Finally, section VI concludes the paper.

\section{DiRECTION FINDING TECHNIQUE}

The principles behind direction finding originate from scintillation based gamma ray detection. When an incident gamma ray is absorbed by the scintillator material, in this a case NaI crystal, a large number of photons are emitted. Further amplification is performed typically by a low-noise photoelectron conversion device such as a photomultiplier tube (PMT). A detector constructed by a single piece of NaI crystal is only capable of detecting and counting gamma rays with no direction identification capability. However, a detection volume composed of three different crystals with independent detection channels can be exploited for directionality [6]. In this work, a spatial configuration of three NaI detectors (D1, D2, D3) have been considered as shown in Figure 1. Noting that each detector is cylindrical with a 2 inch diameter, the counts of incoming gamma rays measured by each detector will be a function of the arrival angle. For instance, if incoming rays are at $60^{\circ}$ arrival angle, D1 will have the highest counts, whereas D2 and D3 will be shadowed by D1 due to its absorption of incident gamma rays at this angle, effectively yielding lower counts in D2 and D3. Similarly, D1 and D2 will have comparable counts while D3 will be shadowed with fewer 


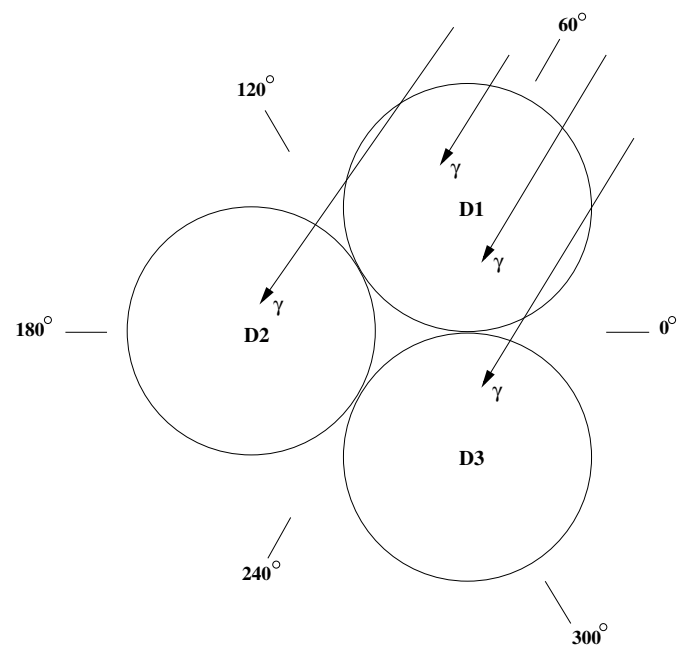

Fig. 1. Top view of the spatial configuration of three cylindrical NaI detectors (D1, D2, D3). For example, D1 will have the highest gamma counts at $60^{\circ}$ arrival angle.

counts for the case of $120^{\circ}$ arrival angle. Thus, based on the absorption/shadowing behavior of this spatial configuration, it is possible to express the activity in each detector for a specific arrival angle as

$$
\alpha_{i}=n_{i} / T, \quad i=1, \ldots, 3
$$

where $\alpha_{i}$ and $n_{i}$ denote the activity and photopeak counts of captured gamma rays in detector $i$ respectively, with $T$ being the total photopeak counts observed in all three detectors. Hence, by gathering statistics for different arrival angles, an empirical yet highly accurate dependency relationship between $\alpha_{i}$ and arrival angle can be created. Consequently, for a given set of observed activities in each detector, the direction of arrival can be determined in a straightforward manner using the gathered statistics.

\section{HARDWARE DESIGN AND IMPLEMENTATION}

The computational sensor architecture is displayed in Figure 2. In support of the aforementioned direction measurement technique, a three-detector $\mathrm{NaI}$ scintillator sensor section with three independent channels has been constructed. These three channels individually interface with a low power sensor analog front end with charge sensitive amplifier, event driven analogto-digital converter (ADC), microcontroller core and its associated peripherals such as memory, I/O hardware, and timers that are integrated in a test chip.

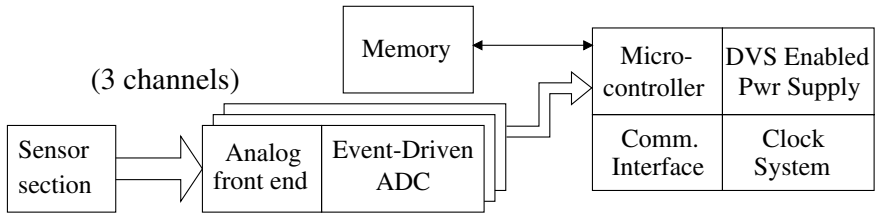

Fig. 2. Directional radiation sensor architecture. The blocks shown following the sensor section comprise the computational radiation sensor and have been included in a test chip using a $0.18 \mu \mathrm{m}$ CMOS technology.

The microcontroller core is responsible for processing and storing the particle events. It is a 16 bit RISC processor design with a peak clock speed of $55 \mathrm{MHz}$ when operating at the maximum voltage of $1.8 \mathrm{~V}$. Also integrated on the same silicon die are resources for various serial communication protocols, timers for timekeeping functions, I/O resources, and system control. In addition, two buck power supplies are integrated on the chip to supply power efficiently to the digital and analog circuitry. The complete design was presented in [5].

\section{A. Sensor Section}

Each detector utilized in the sensor section consists of a $2 \times 2$ inch cylindrical scintillator crystal optically coupled to a 2 inch photomultiplier tube. The 10-stage PMT has a quantum efficiency of $25 \%$ and produces nominally $10^{6}$ current gain with a maximum high voltage bias of $1.1 \mathrm{kV}$, though a lower $850 \mathrm{~V}$ is used with associated lower gain. The assembled crystal and PMT are enclosed in a light-tight housing and $\mu$ metal shield to decrease the effects of environmental magnetic fields on the operation of the PMT. When an incident gamma ray is absorbed by the scintillator, it then emits a large number of photons which are converted into photoelectrons by the photocathode of the photomultiplier tube (PMT). The photoelectrons are amplified by each dynode stage before being collected at the anode to produce the desired signal. The number of photons and therefore the size of the anode current pulse are proportional to the incident gamma ray energy. The detector setup for three channels is shown in Figure 3. The

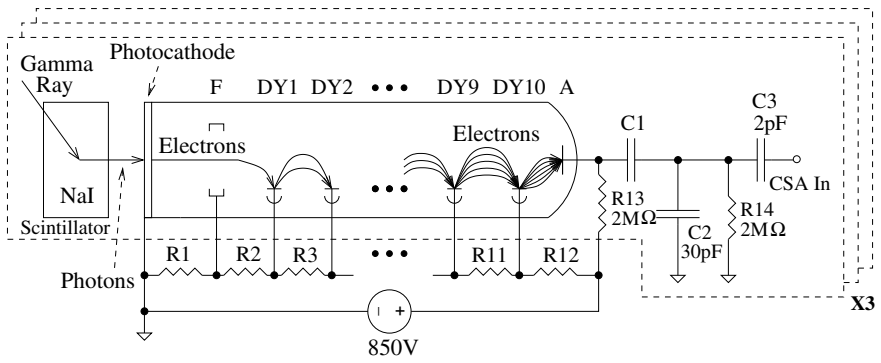

Fig. 3. Three channel NaI scintillator detector section.

three PMTs share a common biasing scheme with the PMTs in a grounded cathode configuration. A charge divider decreases the charge amplitude before going to the charge sensitive amplifier. The resistors R1-R12 form a voltage divider to bias the 10 individual dynodes and focusing electrode, while $\mathrm{C} 1$ is a high voltage DC block capacitor. C2 and C3 form a capacitive charge divider to attenuate the signal so it does not saturate the front end.

\section{B. Complete Directional Gamma Ray Detection Setup}

The directional gamma ray detection setup is shown in Figure 4. The sensor section with three channel $\mathrm{NaI}$ scintillator detectors are housed on a PCB that supports a common biasing scheme with an efficient power supply. The computational radiation sensor section is interfaced to the detector section with another PCB, which is also visible in the figure inset. The complete test setup is connected to a host computer for programming and running tests as well as powering the whole system via the USB interface. 


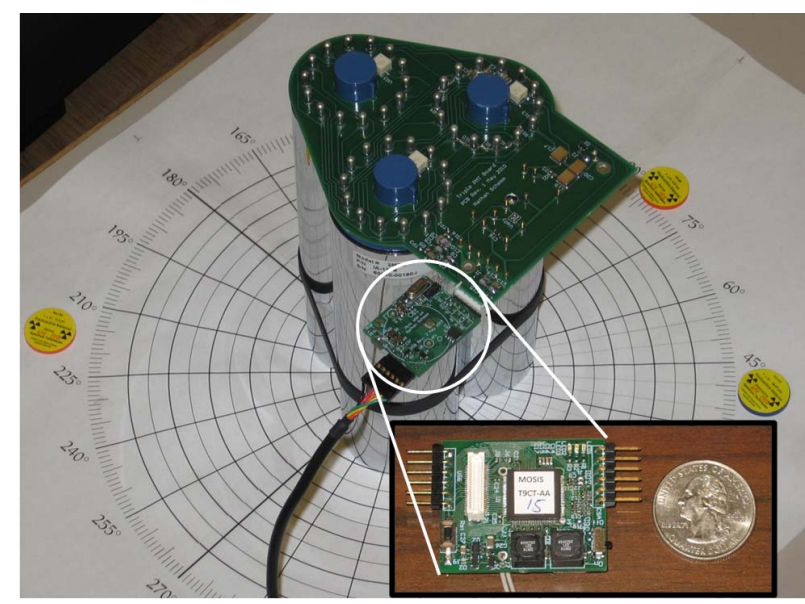

Fig. 4. Directional gamma ray detection test setup displaying gamma sources, the three channel $\mathrm{NaI}$ detector section, and the computational sensor system. The inset shows the photo of the computational sensor section and the fabricated chip.

\section{EMBEDDED SOFTWARE DEVELOPMENT}

The embedded code development to perform directional gamma ray detection consists of two main parts. The first part of the effort focuses on calibration, data collection, and developing the dependency relationship between individual detector activities and the angle of arrival. The second part consists mainly of developing techniques and implementing the associated software code for actual determination of arrival angle based on observed data. These parts are presented next.

\section{A. Data collection}

In this work, the ${ }^{137} \mathrm{Cs}$ isotope that has a single $662 \mathrm{keV}$ photopeak has been considered for the development of algorithms; however, this can be extended to work for other isotopes. Data collection steps are as follows:

- Calibration setup: The relative detection efficiencies of the three detectors have been calibrated by subjecting them to equal gamma ray intensities. As individual $\mathrm{NaI}$ crystals may differ in their detection efficiency, this is a crucial step before collecting data.

- Sweeping the angular position with $5^{\circ}$ increments: In each increment, data is collected from the three channels and processed into pulse height spectra via a simple realtime program that runs on the chip [3]. This effectively implements a multi-channel analyzer. The data collected is then transferred to a host PC for further evaluation. Figure 5 displays a sample capture of the histograms from three detector channels at an angle of $45^{\circ}$. The shadowing of D2 and D3 by D1 is obvious by their photopeak counts.

- Determining the activities in each detector: Next, a peak search algorithm that runs on the host PC identifies the locations and areas of the photoemission peaks present in the histograms. The peak search algorithm is based on well-known methods described in [7]. In this step, the background present in the histogram due to natural sources and Compton scattering is also subtracted. Based on the area calculations of the photoemission peaks, the

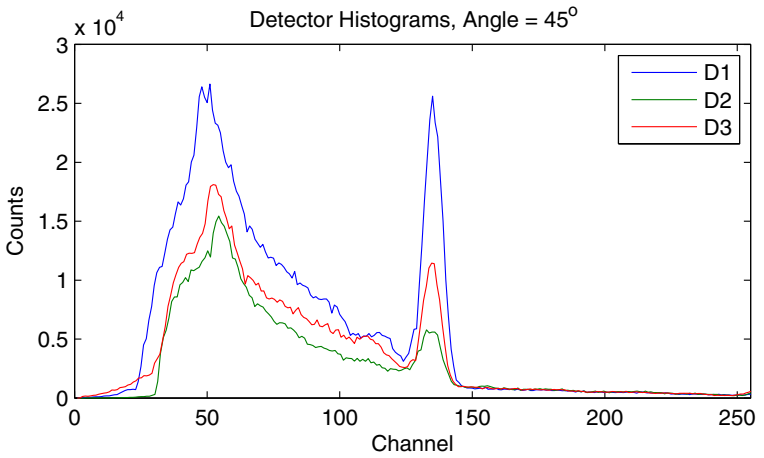

Fig. 5. Pulse height spectra of the three NaI detectors observed at an angle of $45^{\circ}$.

individual activities in each detector $\alpha_{i}$ are determined for each angular position.

- Forming angular position sensitive family of curves: For each detector $\mathrm{D}_{i}, \alpha_{i}$ is stored in the form of a lookup table that is a function of angular position. During this step, the data collected is first smoothed. Figure 6 shows the angular position sensitive family of curves upon conclusion of data collection. Only data from $0-60^{\circ}$ must be stored as a lookup table due to symmetry.

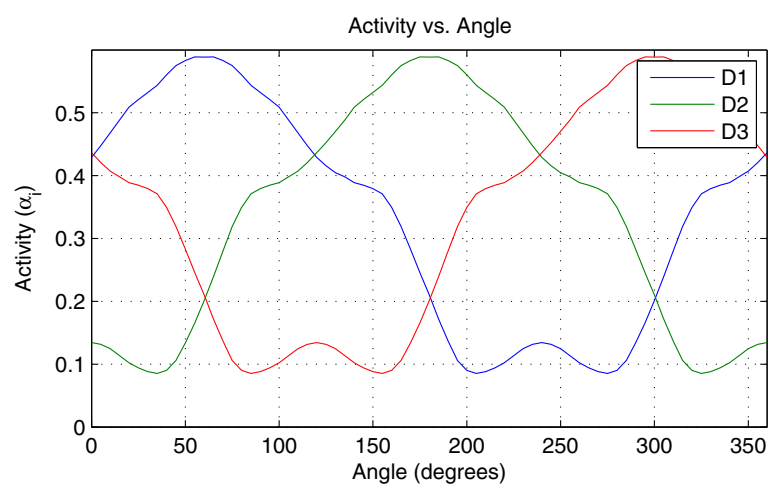

Fig. 6. Angular position sensitive family of activity curves for all the detectors.

\section{B. Implementation}

The following steps are implemented as embedded software code running on the chip for a given direction observation interval.

- Calibration step: The first step is to apply the previously determined calibration coefficients to the gathered histograms to correct for detector efficiency variations.

- Gathering $\alpha_{i}$ : The gathered spectra are then analyzed with respect to their photoemission peak areas which in turn translate to $\alpha_{i}$.

- Search for the angular position: A minimum least squares fit procedure is executed using the observed activities $\alpha_{i}$ and matching it against the lookup table contents. This step is accelerated by decreasing the search angle interval to $60^{\circ}$. This is possible because of the symmetry of the spatial configuration of the detector. 
TABLE I

\section{RESUlts}

The gamma direction algorithm produces an angle measurement after some time of histogram acquisition. The accuracy of the measurement is a strong function of the number of total photopeak counts used in the direction determination. Using the ${ }^{137} \mathrm{Cs}$ source, a single run consists of collecting data for a time period then executing the direction determination to find a single angle. A large number of independent collections were run and the statistics of the resulting angle measurements were computed. The mean of the collected data can be compared with the actual location to determine the accuracy of the system, and the standard deviation is a measurement of the precision. Both the accuracy and standard deviation vary with the angle of incident radiation due to the varying slope of the curves in Figure 6. The maximum accuracy and precision is at $60^{\circ}$ and the minimum is between $0-30^{\circ}$ (These repeat around the circle).

Figure 7 shows the standard of deviation from the sample mean of the system versus photopeak counts for different angles. The $60^{\circ}$ angle is the best case precision, while the $120^{\circ}$ angle is the worst case. These angles repeat, so the precision at $0^{\circ}$ and at $240^{\circ}$ is the same as for $120^{\circ}$.

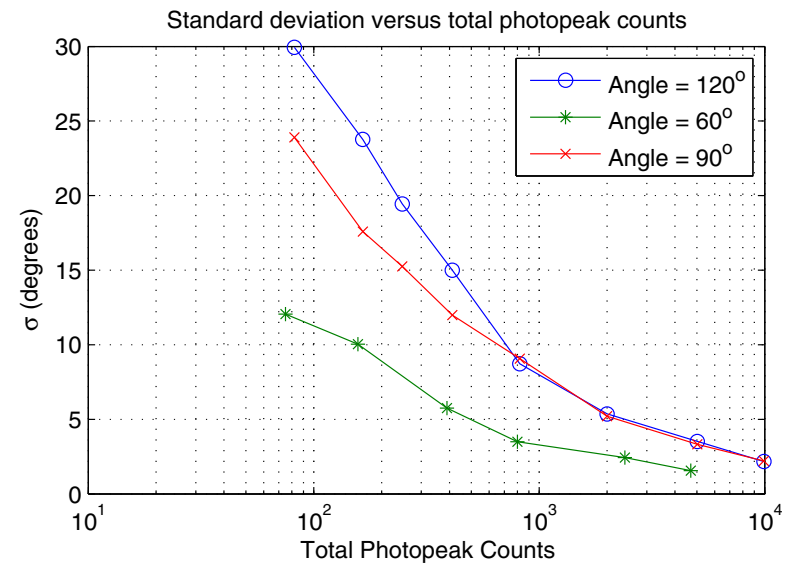

Fig. 7. Standard of Deviation versus Photopeak Counts for ${ }^{137}$ Cs.

For the detector setup with a $1 \mu \mathrm{C}$ source 10 inches from the detector, the $10^{2}$ counts correspond to a 1 second acquisition time.

Table I shows some sample measurements taken after $>10$ minute collection time at which point the variance of the measurement should be small. The errors are due to imperfect calibration of the three detectors. The table also shows how the detector is not affected by other isotopes at different angles which have more energetic gamma rays. These energetic gamma rays also produce Compton peaks in the ${ }^{137} \mathrm{Cs}$ energy range, but they are separated as part of the background in the background estimation step.

Table II gives some specifications of the detector system. The time and execution energy are for processing a single isotope, without gamma isotope identification. To process multiple isotopes, much of the algorithm must be repeated for the different set of ratios.
SAMPLE MEASUREMENTS

\begin{tabular}{|r|c|c|c|}
\hline Description: & $\begin{array}{c}\text { Actual: } \\
\left({ }^{\circ}\right)\end{array}$ & $\begin{array}{c}\text { Meas: } \\
\left({ }^{\circ}\right)\end{array}$ & $\begin{array}{c}\text { Error: } \\
\left({ }^{\circ}\right)\end{array}$ \\
\hline Only ${ }^{137} \mathrm{Cs}$ & 330 & 327 & 3 \\
\hline Only ${ }^{137} \mathrm{Cs}$ & 240 & 245 & 5 \\
\hline Only ${ }^{137} \mathrm{Cs}$ & 60 & 58 & 2 \\
\hline $\begin{array}{r}{ }^{137} \mathrm{Cs},{ }^{133} \mathrm{Ba}, \&{ }^{54} \mathrm{Mn} \text { at } \\
60^{\circ}, 225^{\circ}, 300^{\circ} \text { respectively }\end{array}$ & 60 & 59 & 1 \\
\hline
\end{tabular}

TABLE II

SPECIFICATIONS

\begin{tabular}{|r|l|}
\hline Process: & $0.18 \mu \mathrm{m} \mathrm{CMOS}$ \\
\hline Chip Area: & $13.5 \mathrm{~mm}^{2}$ \\
\hline Max Processor Frequency: & $55 \mathrm{MHz}$ \\
\hline Processor Voltage Active: & $0.9-1.8 \mathrm{~V}$ \\
\hline Processor Active Power: & $150-600 \mu \mathrm{W} / \mathrm{MHz}^{\mathrm{a}}$ \\
\hline Processor Sleep Power: & $2.5-80 \mu \mathrm{W}^{\mathrm{a}}$ \\
\hline Supporting Circuitry Power Consumption: & $3 \mu \mathrm{W}$ \\
\hline Gamma Direction Code Size: & $2.8 \mathrm{kB}$ \\
\hline RAM usage: & $1.5 \mathrm{kB}$ \\
\hline Gamma Direction Execution Time: & $0.88 \mathrm{~ms}^{\mathrm{b}}$ \\
\hline Gamma ID Execution Energy Consumption: & $7.2-29 \mu \mathrm{J}^{\mathrm{a}}$ \\
\hline
\end{tabular}

${ }^{\mathrm{a}}$ Energy/Power varies depending on DVS voltage setting.

${ }^{\mathrm{b}}$ With the processor clock at the maximum of $55 \mathrm{MHz}$.

\section{CONCLUSION}

A low-power gamma direction finding system is presented based on a custom single-chip solution. The simple direction finding algorithm can determine the direction of a particular isotope to within $5^{\circ}$. The system is only sensitive to the desired isotope and can determine the direction in the presence of other undesired isotopes. The present system works with three $\mathrm{NaI}$ scintillators interfaced with three PMTs, however a CsI(Tl) crystal interfaced with photodiodes could be used in future work to decrease the power and size of the detector.

\section{ACKNOWLEDGMENT}

This work was supported by DOE grant "All Solid-State Wireless Sensor Network for Nuclear Proliferation Detection."

\section{REFERENCES}

[1] J. Proffitt, W. Hammond, S. Majewski, V. Popov, R.R. Raylman, A.G. Weisenberger, R. Wojcik, "A Flexible High-rate USB2 Data Acquisition System for PET and SPECT Imaging," IEEE Nucl. Sci. Symp. Conf. 2005, vol. 5, no. 23-29, pp. 2971-2975, Oct. 2005.

[2] M. Schrenk, R. Arlt, P. Beck, H. Boeck, F. Koenig, T. Leitha, "A Real Time, Isotope Identifying Gamma Spectrometer for Monitoring of Pedestrians," IEEE Trans. Nucl. Sci., vol. 52, no. 6, pp. 3012-3019, Dec. 2005.

[3] N. Schemm, S. Balkır, M.W. Hoffman, M Bauer, "A single chip computational sensor system for gamma isotope identification," IEEE Int. Symp. on Cir. and Sys. (ISCAS) 2010, pp. 2271-2274, Paris, June 2010.

[4] N. Schemm, S. Balkır, M.W. Hoffman, "A $4 \mu \mathrm{W}$ CMOS Front End for Particle Detection Applications," Cir. and Sys. II: Express Briefs, IEEE Trans. on, vol. 57, no. 2, pp. 100-104, Feb. 2010.

[5] N. Schemm, S. Balkır, M.W. Hoffman, et al., "A Single Chip Computational Sensor System for Neutron Detection Applications," IEEE Sensors Journal, vol. 10, no. 7, pp. 1226-1233, July 2010.

[6] Y. Shirakawa, T. Yamano, Y. Kobayashi, "Remote Sensing of Nuclear Accidents Using a Direction Finding Detector," 35th Annual Conference of IEEE Industrial Electronics (IECON) 2009, pp. 1917-1922, Porto, Portugal, Nov. 2009.

[7] K. Debertin, R. G. Helmer, Gamma- and X-Ray Spectrometry with Semiconductor Detectors, Elsevier Science Publishers B.V., Amsterdam, North-Holland, 1988. 